\title{
Research on Online Word-of-Mouth Spread Mechanism based on the Node Properties and Word-of-Mouth Type
}

\author{
Xiao-Dong QIAN ${ }^{a,{ }^{*}}$, Min ZHAO ${ }^{b}$, Rui-Dian ZHANG ${ }^{c}$
}

School of Economics and Management, Lanzhou Jiao tong University, Lanzhou 730070, China

aqianxd@Izjtu.mail.com, 'bhaomin8@hotmail.com, c2739275787@qq.com

Key words: Word-of-mouth spread, Complex network, Node properties, Word-of-mouth type.

\begin{abstract}
In this paper, we studied that how node properties and word-of-mouth type influenced the spread of word of mouth. First of all, according to previous research, we embedded all kinds of influencing factors like word-of-mouth type and customer recognition in the word-of-mouth spread model on the basis of SIR virus propagation model. Then, we made a simulation with the word-of-mouth model. According to the simulation, we could draw a graph of node density to examine how these factors affected the change of the node density, which reached a spread mechanism based on the principle and path of word-of-mouth spread. Finally, we give some practical proposals for enterprise marketing.
\end{abstract}

\section{Introduction}

Use the complex network as the framework to study mechanism of word-of-mouth communication. From the point of view of word-of-mouth type, scholars such as Ye [1] have conducted research on online reviews and merchant credibility of China's C2C e-commerce site --- Taobao. The results show good reviews and good business reputation has a positive effect on the increase in sales revenue of merchants. Park [2] (2008) studied the influence of the quality, direction and type of Internet word-of-mouth on the effectiveness of Internet word-of-mouth communication. From the perspective of customer awareness, Li Dongjun [3] proposed the interaction between word of mouth and customer perception from the perspective of customer awareness.

From the perspective of network structure parameters, Styer et al. [4] (2006) studied online forum word-of-mouth communication networks. The distribution of degree degrees obeys the power law distribution rather than the Poisson distribution previously considered.

Yang Yanyang [5] proposed that when the node with high absolute value of recognition has the characteristics of high topology, it has the greatest impact on the network. Li Lin, Sun Jun, and Zhou Zhangjin[6] have studied the heterogeneity of nodes and the difference in network topology to bring about differences in propagation probabilities and propagation ranges.

The SIR model was used as the framework of word-of-mouth communication, embedded in the large-scale online social network-micro blogging data, using MATLAB, Pajek to simulate the word-of-mouth communication.

\section{The Establishment of a Model}

\section{Information Dissemination Model}

The user density is including: $\mathrm{s}(\mathrm{t})$ in susceptible state, $\mathrm{i}(\mathrm{t})$ in infected state, $\mathrm{r}(\mathrm{t})$ in immune state. Due to the highly heterogeneous nature of online networks, online social networks are often abstracted as complex networks.[7]. In general, network information dissemination can be characterized by the SIR model, that is, an infected unknown person can become a communicator with a certain probability and become an immune communicator after a certain period of time [8]. Zhang Haifeng [9] used the virus propagation framework and the connection probabilities between nodes to construct the information propagation model in his research. 


$$
\left\{\begin{array}{l}
\frac{d s_{k}(t)}{d t}=-\lambda k s_{k}(t) \theta_{k}(t)-\gamma s_{k}(t) \\
\frac{d i_{k}(t)}{d t}=\lambda k s_{k}(t) \theta_{k}(t)-i_{k}(t) \\
\frac{d r_{k}(t)}{d t}=i_{k}(t)+\gamma s_{k}(t)
\end{array}\right.
$$

Equation (1) $\theta_{k}(t)$ shows the probability that any given edge of a node of degree $\mathrm{k}$ is connected to an infected node. $s_{k}(t)$ represents the density of susceptible nodes with degree $\mathrm{k}, i_{k}(t)$ represents the density of propagation nodes with degree $\mathrm{k}$, and $r_{k}(t)$ represents the density of immune nodes with degree k. And obey the initial condition as $s_{k}(0)+i_{k}(0)+r_{k}(0)=1, s_{k}(0) \geq 0, i_{k}(0) \geq 0, r_{k}(0) \geq 0$.

\section{Word-of-mouth Communication Model}

\section{Model Construction}

The degree of association $p\left(k^{\prime} / k\right)$ is introduced into the model. The relationship between connection probability and degree is $\theta_{k}(t)=\sum_{k^{\prime}} p\left(k^{\prime} / k\right) i_{k}(t)$.

$$
\left\{\begin{array}{l}
\frac{d s_{k}(t)}{d t}=-\lambda k s_{k}(t) \sum_{k^{\prime}} p\left(k^{\prime} / k\right) i_{k^{\prime}}(t)-\gamma s_{k}(t) \\
\frac{d i_{k}(t)}{d t}=\lambda k s_{k}(t) \sum_{k^{\prime}} p\left(k^{\prime} / k\right) i_{k^{\prime}}(t)-i_{k}(t) \\
\frac{d r_{k}(t)}{d t}=i_{k}(t)+\gamma s_{k}(t)
\end{array}\right.
$$

In equation (2), the infection probability $\lambda$ and the immune probability $\gamma$ are constructed as $\lambda(m)=\mu m /(1+\eta m) \gamma(n)=a-b e^{-\mu m}$.

\section{The Model Framework is Elaborated}

Changes in susceptible nodes are affected by the degree $\mathrm{k}$ of the susceptible nodes themselves, the propagation probability $\lambda$, and their association with the degree of infection $p\left(k^{\prime} / k\right)$.

Changes in immune nodes are mainly affected by the degree of immune node itself $\mathrm{k}$, the immune probability $\gamma$, and its association with the degree of infection $p\left(k^{\prime} / k\right)$.

Change of the infection node is the increase brought about by the growth of susceptible nodes.

\section{The Model Parameter Influence Mechanism is Explained}

(1) Word of mouth type influence mechanism

The word-of-mouth type has a non-linear negative correlation with word-of-mouth communication. Defines the maximum degree of negative word-of-mouth as 0 , and defines the maximum degree of positive as 1 .

(2) Customer recognition influence mechanism

High customer awareness is conducive to word-of-mouth communication. When establishing the propagation probability equation, consider two factors and the state of the surrounding nodes.

$\lambda(m)=\mu m /(1+\eta m) \lambda$ is the probability that a susceptible node becomes an infected node, $\mu$ is a customer awareness, $\eta$ is a word-of-mouth type, and $m$ is the number of neighbor nodes;

$\gamma(n)=a-b \bar{e}^{u n} \gamma$ refers to the probability that an infected node becomes an immune node, a represents the maximum awareness, $b$ refers to the adjustable coefficient, $\mu$ refers to the customer's 
awareness, and n refers to the number of neighbor nodes;

\section{Model Simulation Analysis}

The simulation study adopts the method of controlling variables, and examines the influence of word-of-mouth type and customer cognition on the communication behavior.

\section{Data Acquisition and Analysis}

This article is based on the platform - Sina Weibo's open port API to get the relevant data. Selects 1224 nodes, and uses Pajek software to analyze the complex network diagram as follows in Fig.1:

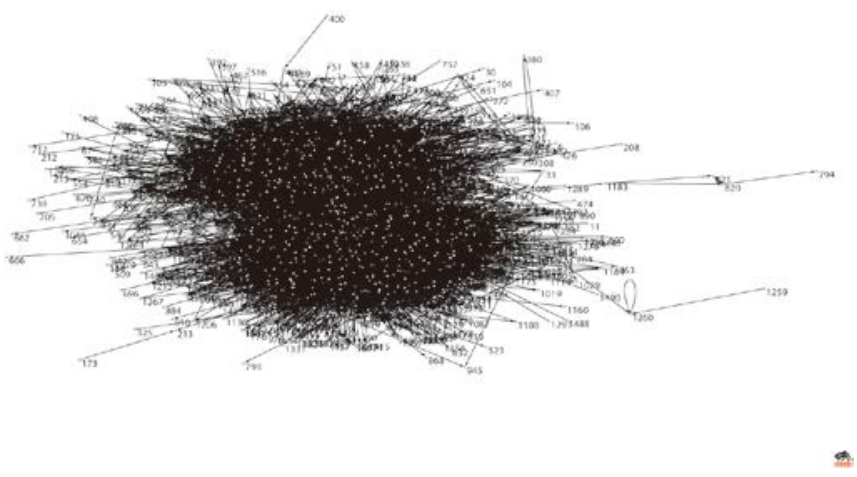

Fig.1 The complex network diagram

The network has a degree of aggregation of 0.1785 and an average degree of 31.0817. The degree of aggregation is not particularly high, and each node is connected to 31 nodes on average.

\section{Analysis of the Overall Evolution Trend of Network Nodes}

The following figure shows the general trend of the three types of nodes. Set the parameter values involved in the impact mechanism. Setting the type of word-of-mouth as the word-of-mouth 0.2 with a relatively large degree of negative influence is more significant. When the customer's cognition value is taken, the average level is 0.4 . And the maximum customer awareness is set to a high degree of 0.8 . The adjustment coefficient is set to 0.4 . Now set the value of the four parameters $\eta=0.2, \mu=0.4, \quad \mathrm{a}=0.8, \mathrm{~b}=0.4$.

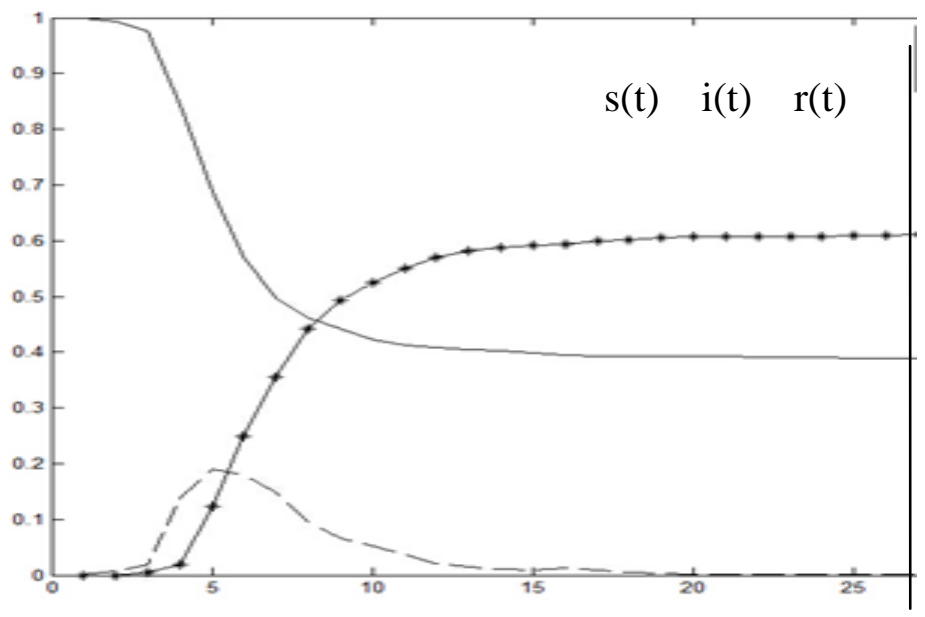

Fig.2 Change of density of different nodes in word-of-mouth communication model

As can be seen from Fig.2, the node curve is not smooth, and the spread of the entire network is not large. Explaining that in the actual network, there are many influencing factors. So companies 
must seize the opportunity to promote the company.

In the initial period, the changes of three types of nodes were particularly significant. In the end, not all nodes are infected. Enterprises can make good use of these network factors and key nodes.

In the initial stage, the nodes in the propagation state increase their number due to the rapid addition of susceptible nodes. The number of infected nodes in the final network becomes zero, and there are only susceptible nodes and immune nodes in the network.

The state of the immune node is completely opposite to that of the susceptible node. The density of immune nodes did not reach 1 , indicating there are nodes that have not received information.

\section{Influence of Word-of-mouth Type on Communication Behavior}

The following figure will describe the influence of different types of word-of-mouth on infected nodes. Control the customer's recognition, change the type of word of mouth, investigate the changing trend of infected nodes.

(1) $\mathrm{a}=0.8, \mathrm{~b}=0.4, \quad \mu=0.4, \quad \eta=0.2$;

(2) $\mathrm{a}=0.8, \mathrm{~b}=0.4, \quad \mu=0.4, \quad \eta=0.4$;

(3) $\mathrm{a}=0.8, \mathrm{~b}=0.4, \mu=0.4, \quad \eta=0.8$.

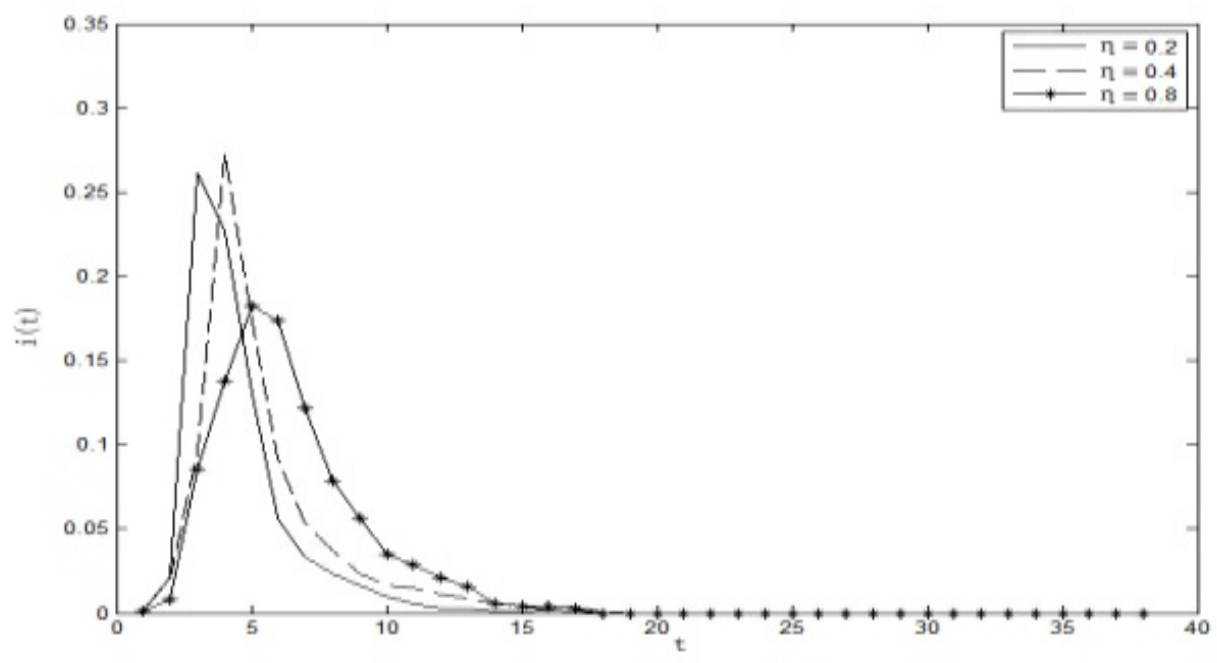

Fig. 3 Infection node density i(t) with trend of word of mouth type

As can be seen from Fig.3, when the type of word of mouth gradually changes from negative to positive, the trend of the entire infection node gradually changes from steep to gentle.

\section{The Influence of Customer Cognition on Communication Behavior}

The picture describes the influence of different degrees of customer awareness on the infection node. Control the type of word of mouth, change the customer's awareness, investigate the changing trend of infected nodes.

(1) $\mathrm{a}=0.8, \mathrm{~b}=0.4, \quad \mu=0.2, \quad \eta=0.2$;

(2) $\mathrm{a}=0.8, \mathrm{~b}=0.4, \mu=0.4, \quad \eta=0.2$;

(3) $\mathrm{a}=0.8, \mathrm{~b}=0.4, \quad \mu=0.8, \quad \eta=0.2$. 


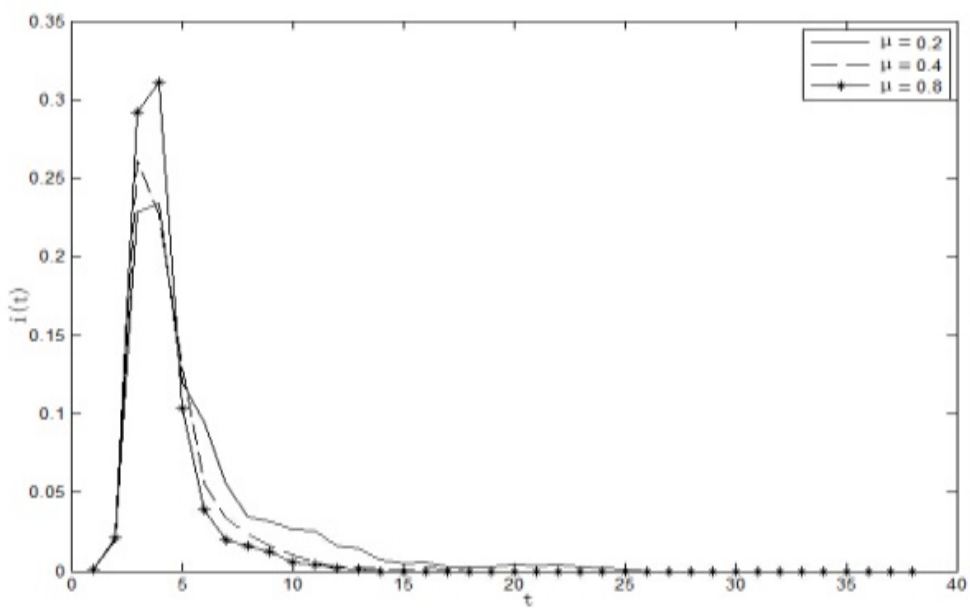

Fig. 4 Trend of infection node density i(t) with customer awareness

As can be seen from Fig.4, first, as the customer's awareness becomes higher and higher, the infection node curve rises more and more steeply, and its peak value continues to increase. Second, the descending trend of the infected node curve becomes steeper, and the higher the awareness is, the more obvious the trend is. When $\mu=0.2$, the infection node curve reaches the peak, there will be a constant peak value for some time.

\section{Conclusions}

The word-of-mouth propagation has the following characteristics: the earlier the density peak appears, the faster word-of-mouth spreads. The spread probability is influenced by the type of word-of-mouth and the customer's cognition. The immune probability is mainly influenced by the customer's cognition. The type of word-of-mouth and the customer's awareness are not completely linear. When companies are doing marketing, they start from three aspects: First, companies must make full use of the network platform to promote enterprise products. Second, companies should appropriately guide the content of word of mouth on companies on the Internet. Third, companies must learn to use the celebrity effect and seize the key nodes in the entire communication network.

\section{Acknowledgement}

This article is supported by National Natural Science Foundation of China under grant No. 71461017.

\section{References}

[1] Qiang Ye, Yijun Li, Melody Kiang, et al. The Impact of Seller Reputation on the Performance of Online Sales: Evidence from TaoBao Buy-It-Now (BIN) Data[J].The DATA BASE for Advances in Information Systems, 2009, 40(1): 12-19.

[2] Park D, Kim S. The Effects of Consumer Knowledge on Message Processing of Electronic Word-of-mouth Via Online Consumer Reviews [J]. Electronic Commerce Research and Applications, 2008, 7(4):339-410.

[3] Dongjun Li. Simulation analysis of word-of-mouth communication effects in complex customer networks [J]. Economy, 2011, 315:116 (In Chinese).

[4] Styer et al. Online Discussion Groups as Social Networks: an Empirical Investigation of Word-of-mouth on the Internet [J]. Journal of Interactive Advertising, 2006, 6 (2): 51-60.

[5] Yanyang Yang et al. Analysis of the stability of complex customer networks [J]. Mathematics in 
Practice and Theory, 2013, 43(23): 113(In Chinese).

[6] Lin Li, Junhua Sun, Zhang Zhou. Service quality word-of-mouth communication based on complex social networks [J]. Systems Engineering, 2009, 6(In Chinese).

[7]Weigang L, Sandes E F O, Zheng J. Querying Dynamic Communities in Online Social Networks[J]. Journal of Zhejiang University SCIENCE C, 2014, 15(2): 81-90.

[8]Zhao X, Wang J. Dynamical Model About Rumor Spreading with Medium[J]. Discrete Dynamics in Nature and Society, 2013(10): 58.

[9] Haifeng Zhang. Dynamic Behavior of SIR Epidemic Model with Immunity on Complex Networks[J].Journal of Shanghai University,2007,13(2):190-191(In Chinese). 